\title{
Perspective: Inverse methods for material design
}

\author{
Avni Jain, ${ }^{1}$ Jonathan A. Bollinger, ${ }^{1}$ and Thomas M. Truskett ${ }^{1,}$ a) \\ McKetta Department of Chemical Engineering, The University of Texas at Austin, Austin, \\ $T X 78712$
}

(Dated: 19 May 2014)

A revised version of this manuscript has been accepted in the AIChE Journal [website link] and can be cited as doi: 10.1002/aic.14491.

\section{Introduction}

Discovery and design of new materials can be conceptualized via the hierarchy shown in Fig. 1, in which engineering performance derives from dominant structural characteristics exhibited at various length scales. Structural features in a material can emerge spontaneously via self- or directed-assembly of primary building blocks (molecules, nanoparticles, colloids, etc.), and they can also be imposed using top-down fabrication techniques. This hierarchical perspective has been enriched by the widespread use of powerful experimental characterization techniques, which provide micro- to mesoscopic information about the morphologies of materials with known macroscopic behaviors. Meanwhile, advances in both simulation methods and computing resources enable the modeling of materials from quantum to continuum scales, offering new opportunities to understand not only how specific structures can form in materials but also how those structures relate to other properties of interest.

Both new materials and new material design rules have been discovered by traversing the hierarchy of Fig. 1 via 'forward' strategies, in which (i) samples with diverse structures are examined to understand how morphology impacts a macroscopic property, or (ii) precursors and synthesis parameters are explored combinatorially to explore the structures that are accessible to a system. That materials with certain periodic structures, such as diamond, can exhibit desirable photonic properties ${ }^{1 / 2}$ or that nanoscale lamellar motifs on the feet of geckos might be mimicked to create dry adhesive materials ${ }^{3}$ represent types of findings that have resulted from the first approach. An example of the second forward strategy is the structural characterization of materials with selfassembling units, such as colloids ${ }^{4}$, quantum $\operatorname{dots}^{5}+7$ or metallic nanoparticles 89 , in which the particle composition and other system parameters are varied to tune the kinetics of assembly and the symmetry of the resulting superlattices 10 .

From a design perspective, however, it is perhaps most natural to begin with a set of macroscopic properties specified by an application and then proceed down the hierarchy of Fig. 1 using 'inverse' strategies to discover which structures, material precursors, and fabrication methods can produce materials consistent with those

\footnotetext{
a) Electronic mail: truskett@che.utexas.edu
}

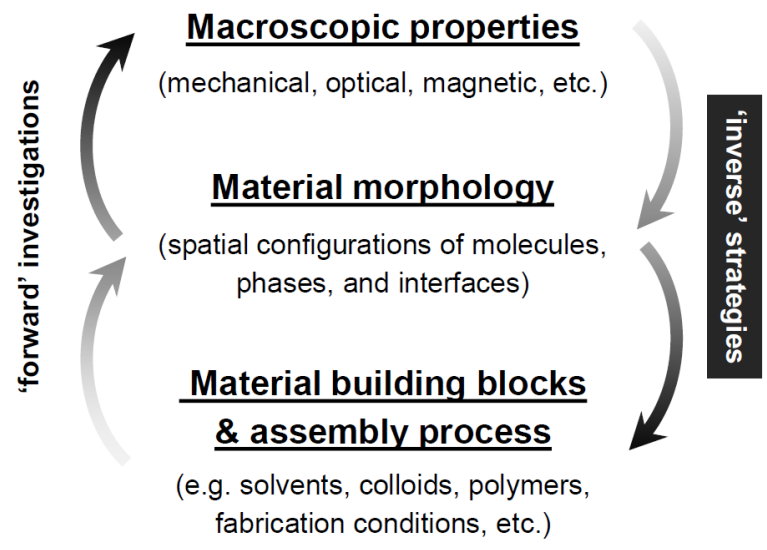

Fig. 1. A hierarchical view of material discovery and design.

specifications. At the coarsest level, such inverse approaches simply ask whether there is a class of morphologies that would be optimal (within known material property constraints) for realizing the specified macroscopic properties. Once optimal morphologies are identified, the focus redirects toward how best to design fabrication or assembly routes with appropriate material precursors for realizing the required structures.

In this Perspective, we highlight several recent studies that illustrate how inverse strategies using appropriate physical models and computational methods can address the following complex materials design questions within the hierarchical framework of Fig. 1.

- Which microstructures reflect-or, alternatively, suppress transmission of-target wavelengths of light for design of structural color or photonic band gap materials, respectively?

- Which 'sparse' template structures direct the assembly of block copolymers into target morphologies for graphoepitaxial nanopatterning applications?

- Which isotropic interactions between colloidal particles promote their self assembly into targeted, open superlattice structures?

We also briefly consider future applications where inverse design methods might return rapid dividends, highlight current limitations of inverse strategies, and speculate 


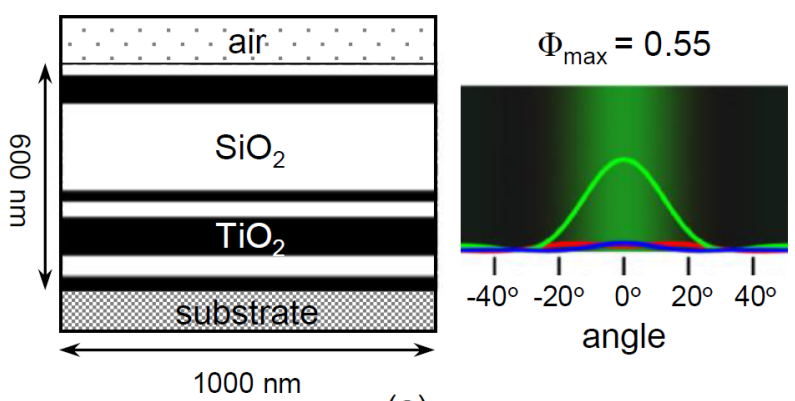

(a)

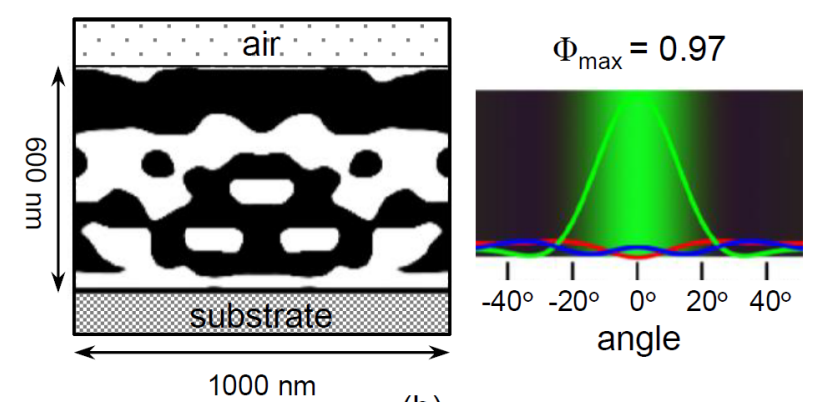

(b)

Fig. 2. Left panels illustrate silica $\left(\mathrm{SiO}_{2}\right)$ and titania $\left(\mathrm{TiO}_{2}\right)$ thin-film morphologies optimized to maximize intensity of a prescribed color (green) in a prescribed direction $\left(0^{\circ}\right)$. Right panels show simulated red-green-blue (RGB) response curves for the designs under exposure to full-spectrum daylight at various incident angles, where $\Phi_{\max }$ is the maximum intensity. In (a), the optimization was constrained to test only alternately layered phases; in (b), this constraint was relaxed. Adapted with permission from 19 .

on how some of the challenges of this field might be addressed.

\section{Designing structures for target properties}

Dielectric thin-film morphologies for structural color applications. Numerical methods, led by finite-element based topology optimization (TO), have been used since the late 1980 s to determine material microstructures that meet design specifications ${ }^{[3]}$ Initially, these methods were used to design heterogeneous or multiphase materials with targeted thermal expansivity $\sqrt{14 \mid 15}$ as well as elastic ${ }^{16}$ and piezoelectric ${ }^{17}$ properties. During the past decade, however, the TO algorithm has been extended to discover new microstructures consistent with desired acoustic $^{[18}$, nanophotonic ${ }^{19}$, and photovoltaic ${ }^{20}$ behaviors as well. Non-linear constrained optimizations have also been employed to design surface textures of thin film solar cells for enhanced photon absorption ${ }^{21}$ and to discover novel amorphous photonic structures22.

Inspired by the vibrantly hued yet non-pigmented scales of Morpho butterflies 2324 and other insects, in which the apparent colors arise from the interaction of light with the wings' nanoscopic cuticle-air features, researchers recently used TO to control perceived color solely by manipulating the material architecture ${ }^{19}$
Specifically, the authors optimized thin-film dielectric morphologies to produce specific and high-intensity structural color responses; i.e., without the use of individual pigments. In Fig. 2, the left-hand panels illustrate silica $\left(\mathrm{SiO}_{2}\right)$ and titania $\left(\mathrm{TiO}_{2}\right)$ dielectric films with nanostructural features optimized for a green response on exposure to daylight at a specular angle of $0^{\circ}$. Two design optimizations were explored. Fig. 2(a) shows the result when the dielectric material was required to be alternately layered (i.e., one-dimensional gratings), and Fig. 2(b) shows the more complex structure of the unconstrained optimum. The right-hand panels provide the red-green-blue (RGB) response curves over a wider angular spectrum with the background displaying the perceived color. While both the layered and disordered morphologies meet the set objective, the intensity is clearly optimized by the disorganized microstructure.

More broadly, it was demonstrated that the qualitative structural features of the optimal films depend sensitively on the desired color response in a way that could not be guessed a priori without theoretical guidance. Designs for a wide range of colors were presented, and for some wavelengths even the unconstrained optimizations surprisingly found layered morphologies that were optimal19. For angle-dependent (iridescent) color responses, entirely different microstructures are required with the details depending on the manufacturing constraints. In short, this theoretical strategy, rooted in deeply-established physics principles 26 28, provides an example of how inverse methods can make very specific and non-trivial theoretical predictions about the classes of microstructures that should be tested and possibly pursued in the next stage of experimental design.

Disordered materials with complete photonic band gaps. A photonic band gap (PBG) is a frequency range over which transmission of incident electromagnetic waves is blocked. By combining at least two material components with disparate dielectric constants in specific ratios and configurations, the transmission of light frequencies can be blocked for some or all incident directions and polarizations, the latter corresponding to a 'complete' PBG.

Both forward and inverse studies of photonic materials have discovered periodic microstructural configurations that exhibit PBG\$ ${ }^{29}$, including diamond ${ }^{11}$ and woodpile structures ${ }^{2}$, and cylindrical packings in hexagona $3{ }^{30}$ and honeycomb ${ }^{31}$ patterns. Beyond periodic structures, a developing research area considers how light interacts with disordered and nonperiodic structures ${ }^{32}$. Examples include photonic glasses ${ }^{33}$, display of structural colors in animals 24 , and packing of avian photoreceptors 34 . Despite the interest in disordered photonic materials, broad principles for designing disordered PGB microstructures have been slow to emerge.

A recent inverse design study ${ }^{22}$ focused on discovering novel microstructures consisting of irregular 2D point patterns that exhibit complete PBGs. To make the optimization tractable, the authors constrained their design space to 'hyperuniform' structures only ${ }^{35 / 36}$, which ex- 


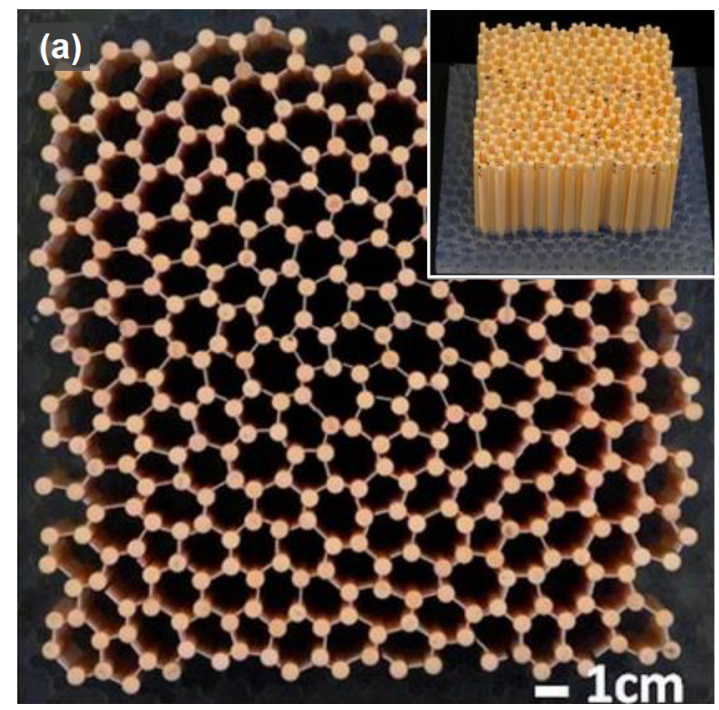

(b) TM polarization

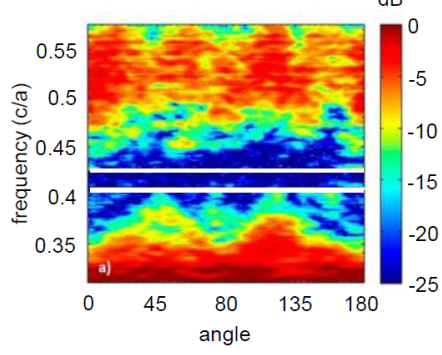

(c) TE polarization

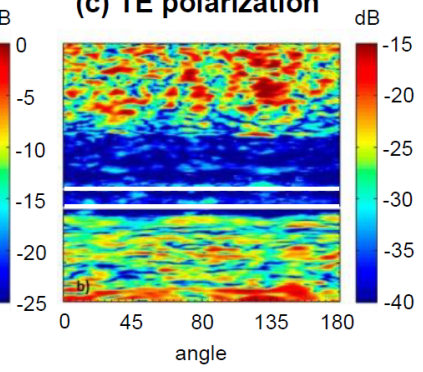

Fig. 3. (a) A fabricated amorphous 2D pattern comprising alumina cylinders and connective sheets, designed to display a complete photonic band gap (PBG) (inset shows a side view of the structure). The lower panels show measured transmission strength (in $\mathrm{dB}$ ) for various light frequencies and incident angles. The PBGs in response to (b) transverse magnetic (TM) and (c) transverse electric (TE) polarizations are bounded by white lines. Adapted with permission from ${ }^{22}$.

hibit suppressed density fluctuations (all crystals, quasicrystals and a subset of disordered patterns-e.g., those corresponding to maximally random jammed particle packings ${ }^{37}$ are hyperuniform). Using pre-generated hyperuniform point patterns to position alumina cylinders, the authors numerically optimized the common cylinder radius to maximize the PBG frequency range. Fig. 3 illustrates a fabricated version of the optimal 2D pattern and the measured PBGs, which matched the theoretical predictions. Most importantly, the authors were able to quantify common structural characteristics of optimal hyperuniform 2D structures for PBG materials, including their enhanced short-ranged geometric order (as characterized by the strength of cylinder-cylinder spatial correlations) and the uniformity of their local topology (as measured by the cylinder coordination number). The combination of these characteristics-suppressed density fluctuations, short-range correlations, and uniform topology-provides a new structural 'design rule' discovered by inverse methods that has more recently been used to generate, using Direct Laser Writing techniques 38 40, synthetic disordered 3D photonic structures yielding complete PBGs 41 .

Designing directed- and self-assembly processes for target structures

Once desirable target structures are identified, the challenge of how to synthesize materials with the required morphologies remains. Broadly speaking, there are two types of approaches: (1) top-down fabrication and (2) bottom-up assembly. Top-down approaches typically use pattern transfer, etching, or deposition technologies to impose the desired structural features on the materials of interest 4247 . Assembly, on the other hand, relies upon spontaneous formation of target morphologies, which can be driven by 'self' interactions between the primary units or building blocks of the material system or additionally 'directed' by carefully chosen external fields or boundaries in the system $\frac{48,50}{5}$. In many applications, both top-down and bottom-up strategies play a role. For example, directed assembly often relies upon top-down fabrication methods like lithography to create an initial template (e.g., a chemically or topographically prepatterned substrate) that helps to steer the assembly of smaller primary units (e.g., block copolymers) into a desired structure $51 \sqrt[54]{54}$.

From a fundamental perspective, directed- and selfassembly methods pose challenging questions about how best to choose system parameters and material components in order to promote organization of a condensed matter system into a target structure instead of a competing morphology. As we describe below, inverse methods for assembly can take advantage of multidimensional optimization methods and statistical mechanical theories of complex fluids, and thus they are natural tools to help address these questions.

Templates for directed assembly of block-copolymer (BCP) morphologies. As a first step, templates for directed assembly are typically designed by intuition: imprinting physical or chemical patches along regions where complementary phases are to be located. A key challenge is to use inverse design theory to develop templates with minimum feature density and complexity while still inducing assembly of structures consistent with device-pattern specifications 55156 . A recent series of studies 57 [59] used Monte-Carlo (MC) based optimizations to design substrates with maximally sparse patterns that promote target BCP phase-separated patterns, including technologically-relevant features like bends, junctions, and terminations. The templates consisted of posts selectively attracted to one of the $\mathrm{BCP}$ phases situated on a phase-neutral substrate. Fig. 4 presents the authors' inverse workflow, in which they (a) specified the target BCP morphology and (b) optimized the post configuration (shown as dots) based on predictions from self-consistent field theory (SCFT). They then (c) 
(a) Target morphology

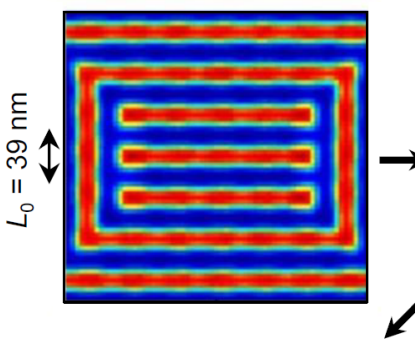

(c) Fabricated template

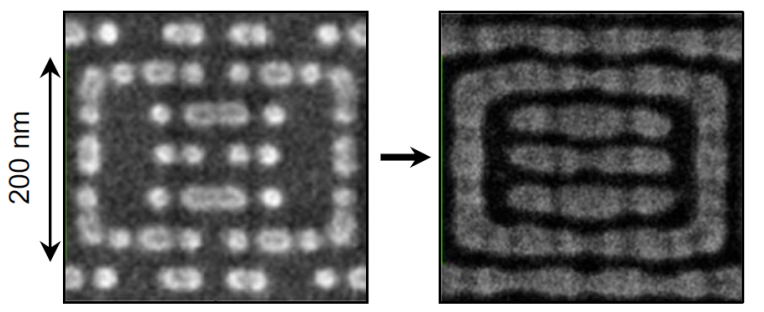

(b) SCFT validation

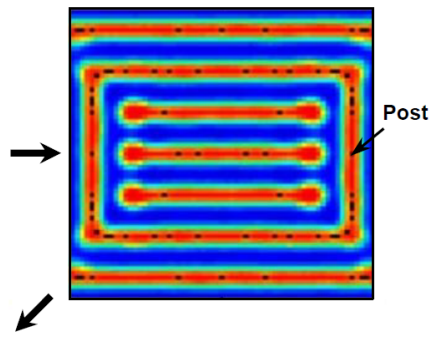

(d) BCP assembly
Fig. 4. (a) Target polystyrene-b-polydimethylsiloxane (PS-b-PDMS) block-copolymer (BCP) morphology (minority PDMS regions are red, majority PS regions are blue). (b) Simulation results confirming the target structure is displayed using the inversely optimized template of posts (shown as black dots). (c) The optimal template, fabricated via electron beam lithography. (d) SEM scan of the resulting BCP morphology. Adapted with permission from 58 .

fabricated the template using electron beam lithography and (d) demonstrated that the target polystyreneb-polydimethylsiloxane (PS-b-PDMS) BCP morphology was indeed assembled in the optimized post configuration. As an alternative to the MC-based inverse strategy, other researchers ${ }^{60}$ designed similar BCP templates using an evolutionary algorithm based on Cahn-Hillard equations for non-equilibrium phase separation. While their optimal templates have yet to be validated experimentally, the authors emphasized that using analytical theories should make increasingly complex design problems computationally tractable.

Designing interactions for targeted superlattice selfassembly. An important focus of the modern selfassembly literature has been on understanding which equilibrium superlattice phases are formed by various nano- or microscale colloids $\sqrt{61}$ based on their shape $e^{62,67}$, size ${ }^{[2|68| 69}$, surface texture ${ }^{70}$, surface coating 71 , 73 , particle-solvent interactions ${ }^{10}$, etc. A particularly fruitful topic has been the examination of assembly driven by particle-surface functionalizations which provide orientation-specific interparticle interactions ${ }^{74}+76$ that mimic atomic covalent bonding via, e.g., DNA sticky ends $77 \sqrt{80}$ or similar interactions promoted by complementary inorganic ligands $\$ 7181 \mid 82$.

However, outstanding challenges remain in characterizing the effective interactions between suspended nano-(or colloidal) particles as well as in elucidating their stable equilibrium phases. A key challenge is to identify the basic limitations that interaction isotropy between particles places on the types of achievable periodic structures. This is important because there are practical advantages in terms of the synthesis and assembly kinetics of nano (or colloidal) particles with approximately isotropic (versus orientation-dependent or "patchy") interactions 83 .

Statistical-mechanics based computational studies 84,88 have demonstrated that isotropic interactions can drive assembly of exotic, low-coordinated 2D (e.g. Kagome, honeycomb, snub-square) and 3D (e.g. diamond, simple cubic) lattices, which represents a break from the conventional understanding that directional interactions are necessary to self-assemble such open lattice structures. Recently $\sqrt[85]{ }$, authors have used inverse statisticalmechanical optimization methods to design pairwise interaction potentials that maximized the phase diagram footprints (i.e. density ranges) over which specific 3D lattice, e.g. diamond, ground states were favored. In this study, the potentials were constrained to be shortrange and convex-repulsive, features qualitatively similar to measured interactions between particles uniformly grafted with ligands interacting in a solvent medium 8990 . Using free-energy based MC methods ${ }^{91}$, the associated superlattice phase diagrams were calculated (see Fig. 5) and target phases based on the designed potentials were found to be robust to variations in the osmotic secondvirial coefficient of the interparticle interaction (i.e, solvent quality, analogous to temperature in these systems).

While these inverse strategies can provide precise mathematical interactions that drive assembly to target structures, realizing these effective potentials in real systems remains an open challenge 829495 . Certainly, experts in colloidal particle synthesis can attempt to creatively intuit the precursor materials and fabrication conditions that result in approximations of optimal effective potentials, though at present this represents a rather open-ended enterprise. A more insightful line of inquiry might be to optimize interaction models composed explicitly of experimentally-controllable parameters (e.g. colloidal core material, particle size, grafted ligand lengths, graft densities, etc.). In one such investigation $\frac{96}{\text {, re- }}$ searchers used genetic algorithms to design the density and length of complementary sticky ssDNA strands to be grafted on spherical colloids to form target crystal structures. They based their optimization on a previously established numerical mode ${ }^{97}$ and thus, were able to replicate experimental observations of crystalline assembly for similar DNA-grafted colloidal systems 57 . Unfortunately, few existing models capture the overarching physics of colloidal interactions over accessible design spaces while remaining tractable for optimization search routines. $\frac{98}{100}$ Overcoming this knowledge gap necessitates a return to theoretical work in complex fluids, focused on establishing accurate analytical and numerically tractable models that reflect nano- and microscale 


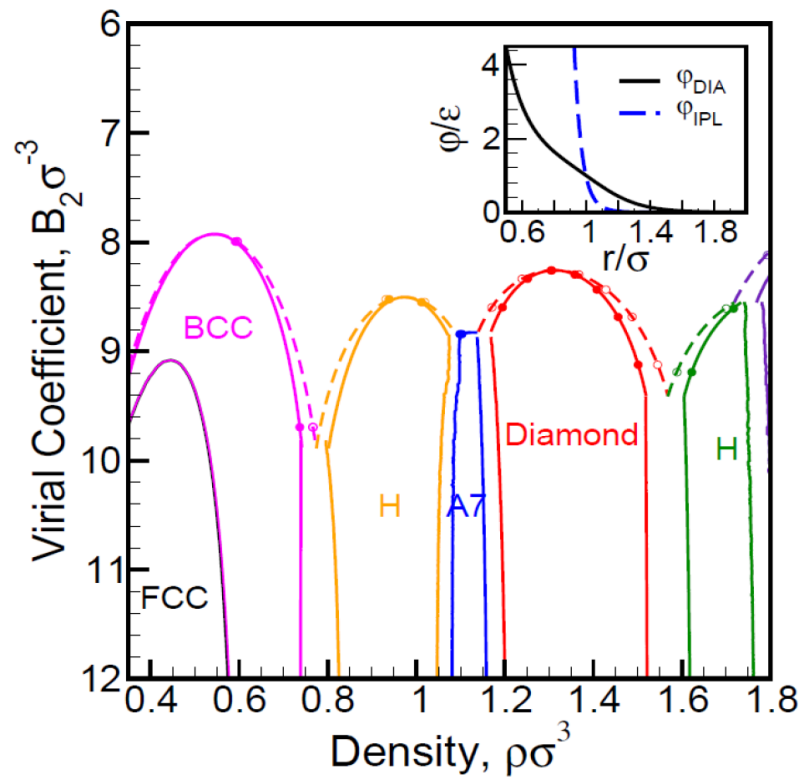

Fig. 5. Superlattice-fluid phase diagram of particles with an isotropic interparticle potential optimized to exhibit a diamond crystal ground-state over the widest possible density range. The inset shows the diamond-forming potential $\left(\varphi_{\text {DIA }}\right)$ compared against an inverse power law $(\mathrm{n}=12)$ potential $\left(\varphi_{\text {IPL }}\right)$, which highlights the main feature of the optimized potential: its softer repulsion. Coexistence lines were obtained via free-energy MC simulation methods from 91 93.

colloidal physics.

\section{Future directions}

Beyond the highlighted studies above, there are several emerging technological contexts in which computational inverse strategies are already being used, and others where they might return quick dividends. These endeavors include studies focused on optimizing solar cell domain distributions for increased photovoltaic efficiency 21, mesoscale architectures composed of nanocrystals for enhanced energy flow 101 ; dopant concentrations in polymer and inorganic matrices for target functionalities ${ }^{102}$; multiphase interfaces for thermoelectric transport ${ }^{103}$; external field $\$ 104$ and interparticle interactions $\sqrt{105}$ for enhanced single-particle mobility; and faceted colloids that form target periodic structures and tesselations 64|106. Inverse strategies should also prove useful in optimizing new fabrication approaches, e.g., identifying ideal blade micro-features for controlling thinfilm organic-semiconductor crystals at industrial manufacturing scales $107 / 108$, and also offer the opportunity to revisit long-standing physical questions, including the evolution of granular shapes that possess target macroscopic stress responses $\frac{109}{}$.

It cannot be overemphasized that along with advances in computing capabilities and numerical tools 110 114, the utility of inverse strategies relies on the physical accuracy of the underlying hierarchical connections between properties, structure, and assembly. As illustrated in this Perspective, many links between macroscopic properties, e.g. mechanical and photonic properties, and underlying microstructures are well understood and have been exploited to design new materials and establish new design rules. Also, as device properties are increasingly understood to emerge from material attributes at the nanoscale and smaller, continuum and classical descriptions of materials may reach their fundamental thresholds of applicability. Going forward, their validity needs to be established across multiple lengthscales, and, if necessary, new integrated multiscale connections must be developed. Such challenges are already apparent in terms of holistically understanding the physics of assembly, as exemplified in our discussion of the inverse design of self-assembling colloids. And while significant knowledge gains are being made through extensive forward-strategy investigations, their successful integration into inverse methods depends on distilling and validating computationally tractable physical theories.

We note that the hierarchy of Fig. 1 is a coarse simplification, meant to encompass the broadest possible collection of contemporary material design problems. It may not always be reasonable or advantageous to pose inverse design problems that separately address either Property $\rightarrow$ Structure or Structure $\rightarrow$ Assembly connections in isolation. Especially when macroscopic material properties can be related to the chemical make-up of the material precursors, it may be possible to tune the precursors directly for the desired property, without explicitly specifying structural connections.

The physical models and problem constraints, i.e. 'user inputs', that must be chosen while formulating the inverse strategies are ultimately validated when the outcomes of experimental synthesis and characterization match promising solutions. When outcomes do not match the theoretical predictions, however, there are still gains to be made. Forward investigations-whether conducted via experiments or simulations-can be used to clarify system responses within regions of the design space where predictions failed, and the underlying theoretical connections can be revised in light of new experimental findings. This feedback loop between theory and experiments can iteratively provide novel and non-intuitive physical insights that bridge different levels of the material design hierarchy. Subsequently, these new connections can streamline the development of an expanding portfolio of technologically-relevant materials, from desired device properties down to the choice of modular and inexpensive material precursors.

\section{Acknowledgments}

The authors gratefully acknowledge support from the Robert A. Welch Foundation (F-1696), the National Science Foundation (CBET-1065357), and the Gulf of Mexico Research Initiative.

\footnotetext{
${ }^{1}$ K. M. Ho, C. T. Chan, and C. M. Soukoulis, "Existence of a
} 
photonic gap in periodic dielectric structures," Physical Review Letters, vol. 65, no. 25, pp. 3152-3155, 1990.

${ }^{2} \mathrm{M}$. Maldovan and E. Thomas, "Diamond-structured photonic crystals," Nature Materials, vol. 3, pp. 593-600, SEP 2004.

${ }^{3}$ A. Geim, S. Dubonos, I. Grigorieva, K. Novoselov, A. Zhukov, and S. Shapoval, "Microfabricated adhesive mimicking gecko foot-hair," Nature Materials, vol. 2, pp. 461-463, JUL 2003.

${ }^{4}$ D. Vanmaekelbergh, "Self-assembly of colloidal nanocrystals as route to novel classes of nanostructured materials," Nano Today, vol. 6, no. 4, pp. 419-437, 2011.

${ }^{5}$ H.-Y. Tuan, D. C. Lee, and B. A. Korgel, "Nanocrystalmediated crystallization of silicon and germanium nanowires in organic solvents: The role of catalysis and solid-phase seeding," Angewandte Chemie, vol. 118, no. 31, pp. 5308-5311, 2006.

${ }^{6}$ C. M. Evans, L. C. Cass, K. E. Knowles, D. B. Tice, R. P. H. Chang, and E. A. Weiss, "Review of the synthesis and properties of colloidal quantum dots: the evolving role of coordinating surface ligands," Journal of Coordination Chemistry, vol. 65, no. 13, pp. 2391-2414, 2012.

${ }^{7}$ Y. Yu, C. A. Bosoy, C. M. Hessel, D.-M. Smilgies, and B. A. Korgel, "Silicon nanocrystal superlattices," ChemPhysChem, vol. 14 , no. 1 , pp. 84-87, 2013.

${ }^{8}$ S. I. Stoeva, B. L. V. Prasad, S. Uma, P. K. Stoimenov, V. Zaikovski, C. M. Sorensen, and K. J. Klabunde, "Facecentered cubic and hexagonal closed-packed nanocrystal superlattices of gold nanoparticles prepared by different methods," The Journal of Physical Chemistry B, vol. 107, no. 30, pp. 74417448, 2003.

${ }^{9}$ M. R. Rasch, E. Rossinyol, J. L. Hueso, B. W. Goodfellow, J. Arbiol, and B. A. Korgel, "Hydrophobic gold nanoparticle self-assembly with phosphatidylcholine lipid: Membrane-loaded and Janus vesicles," Nano Letters, vol. 10, no. 9, pp. 3733-3739, 2010.

${ }^{10}$ A. Courty, J. Richardi, P.-A. Albouy, and M.-P. Pileni, "How to control the crystalline structure of supracrystals of 5-nm silver nanocrystals," Chemistry of Materials, vol. 23, no. 18, pp. 41864192, 2011.

${ }^{11}$ K. Bian, J. J. Choi, A. Kaushik, P. Clancy, D.-M. Smilgies, and T. Hanrath, "Shape-anisotropy driven symmetry transformations in nanocrystal superlattice polymorphs," ACS Nano, vol. 5, no. 4, pp. 2815-2823, 2011.

${ }^{12}$ M. J. Pavan, E. Ploshnik, and R. Shenhar, "Nanoparticle assembly on topographical polymer templates: Effects of spin rate, nanoparticle size, ligand, and concentration," The Journal of Physical Chemistry B, vol. 116, no. 47, pp. 13922-13931, 2012.

${ }^{13} \mathrm{O}$. Sigmund and K. Maute, "Topology optimization approaches A comparative review," Structural And Multidisciplinary Optimization, vol. 48, pp. 1031-1055, DEC 2013.

${ }^{14} \mathrm{O}$. Sigmund and S. Torquato, "Composites with extremal thermal expansion coefficients," Applied Physics Letters, vol. 69, pp. 3203-3205, NOV 181996.

${ }^{15} \mathrm{O}$. Sigmund and S. Torquato, "Design of materials with extreme thermal expansion using a three-phase topology optimization method," Journal of the Mechanics and Physics of Solids, vol. 45, pp. 1037-1067, JUN 1997.

${ }^{16} \mathrm{O}$. Sigmund, "Tailoring materials with prescribed elastic properties," Mechanics of Materials, vol. 20, no. 4, pp. $351-368$, 1995.

${ }^{17}$ O. Sigmund, S. Torquato, and I. Aksay, "On the design of 1-3 piezocomposites using topology optimization," Journal of Materials Research, vol. 13, pp. 1038-1048, APR 1998.

${ }^{18}$ M. B. Duhring, J. S. Jensen, and O. Sigmund, "Acoustic design by topology optimization," Journal of Sounds and Vibration, vol. 317, pp. 557-575, NOV 112008.

${ }^{19}$ J. Andkjaer, V. E. Johansen, K. S. Friis, and O. Sigmund, "Inverse design of nanostructured surfaces for color effects," Journal of the Optical Society of America B-Optical Physics, vol. 31, pp. 164-174, JAN 2014.

${ }^{20}$ M. B. Duhring and O. Sigmund, "Optimization of extraordinary optical absorption in plasmonic and dielectric structures,"
Journal of the Optical Society of America B-Optical Physics, vol. 30, pp. 1154-1160, MAY 2013.

${ }^{21}$ X. Sheng, S. G. Johnson, J. Michel, and L. C. Kimerling, "Optimization-based design of surface textures for thin-film si solar cells," Opt. Express, vol. 19, pp. A841-A850, Jul 2011.

${ }^{22}$ W. Man, M. Florescu, E. P. Williamson, Y. He, S. R. Hashemizad, B. Y. C. Leung, D. R. Liner, S. Torquato, P. M. Chaikin, and P. J. Steinhardt, "Isotropic band gaps and freeform waveguides observed in hyperuniform disordered photonic solids," Proceedings of the National Academy of Sciences of the United States of America, vol. 110, pp. 15886-15891, OCT 12013.

${ }^{23}$ A. Saito, "Material design and structural color inspired by biomimetic approach," Science 83 Technology of Advanced Materials, vol. 12, DEC 2011.

${ }^{24}$ F. P. Barrows and M. H. Bart, "Photonic Structures in Biology: A Possible Blueprint for Nanotechnology," Nanomaterials and Nanotechnology, vol. 4, JAN 282014.

${ }^{25} \mathrm{P}$. Vukusic, B. Hallam, and J. Noyes, "Brilliant whiteness in ultrathin beetle scales," Science, vol. 315, p. 348, JAN 192007.

${ }^{26}$ G. Bao, D. C. Dobson, and J. A. Cox, "Mathematical studies in rigorous grating theory," J. Opt. Soc. Am. A, vol. 12, pp. 10291042, May 1995.

${ }^{27} \mathrm{G}$. Bao, "Finite element approximation of time harmonic waves in periodic structures," SIAM Journal on Numerical Analysis, vol. 32, no. 4, pp. 1155-1169, 1995.

${ }^{28}$ K. Dossou, M. A. Byrne, and L. C. Botten, "Finite element computation of grating scattering matrices and application to photonic crystal band calculations," Journal of Computational Physics, vol. 219, no. 1, pp. 120 - 143, 2006.

${ }^{29}$ C. Soukoulis, Photonic Crystals and Light Localization in the 21st Century. NATO science series: Mathematical and physical sciences, Springer Netherlands, 2001.

${ }^{30}$ D. Cassagne, C. Jouanin, and D. Bertho, "Hexagonal photonicband-gap structures," Phys. Rev. B, vol. 53, pp. 7134-7142, Mar 1996.

${ }^{31}$ O. Sigmund and K. Hougaard, "Geometric properties of optimal photonic crystals," Physical Review Letters, vol. 100, p. 153904, Apr 2008.

${ }^{32}$ D. S. Wiersma, "Disordered photonics," Nature Photonics, vol. 7, pp. 188-196, MAR 2013.

${ }^{33}$ P. Garcìa, R. Sapienza, À. Blanco, and C. Lòpez, "Photonic glass: A novel random material for light," Advanced Materials, vol. 19, no. 18, pp. 2597-2602, 2007.

${ }^{34}$ Y. Jiao, T. Lau, H. Hatzikirou, M. Meyer-Hermann, J. C. Corbo, and S. Torquato, "Avian photoreceptor patterns represent a disordered hyperuniform solution to a multiscale packing problem," Phys. Rev. E, vol. 89, p. 022721, Feb 2014.

${ }^{35} \mathrm{~S}$. Torquato and F. H. Stillinger, "Local density fluctuations, hyperuniformity, and order metrics," Phys. Rev. E, vol. 68, p. 041113 , Oct 2003.

${ }^{36}$ M. Florescu, S. Torquato, and P. J. Steinhardt, "Complete band gaps in two-dimensional photonic quasicrystals," Phys. Rev. B, vol. 80, p. 155112 , Oct 2009.

${ }^{37}$ S. Torquato, T. M. Truskett, and P. G. Debenedetti, "Is random close packing of spheres well defined?," Phys. Rev. Lett., vol. 84, pp. 2064-2067, Mar 2000.

${ }^{38}$ M. Deubel, G. Von Freymann, M. Wegener, S. Pereira, K. Busch, and C. M. Soukoulis, "Direct laser writing of three-dimensional photonic-crystal templates for telecommunications," Nature materials, vol. 3, no. 7, pp. 444-447, 2004.

${ }^{39}$ J. Haberko and F. Scheffold, "Fabrication of mesoscale polymeric templates for three-dimensional disordered photonic materials," Opt. Express, vol. 21, pp. 1057-1065, Jan 2013.

${ }^{40}$ N. Muller, J. Haberko, C. Marichy, and F. Scheffold, "Silicon hyperuniform disordered photonic materials with a pronounced gap in the shortwave infrared," Advanced Optical Materials, vol. 2, no. 2, pp. 115-119, 2014.

${ }^{41}$ S. F. Liew, J.-K. Yang, H. Noh, C. F. Schreck, E. R. Dufresne, C. S. O'Hern, and H. Cao, "Photonic band gaps in threedimensional network structures with short-range order," Phys. 
Rev. A, vol. 84, p. 063818, Dec 2011.

${ }^{42} \mathrm{M}$. Geissler and Y. Xia, "Patterning: Principles and some new developments," Advanced Materials, vol. 16, no. 15, pp. 12491269, 2004.

${ }^{43}$ A. Biswas, I. S. Bayer, A. S. Biris, T. Wang, E. Dervishi, and F. Faupel, "Advances in topdown and bottomup surface nanofabrication: Techniques, applications \& future prospects," Advances in Colloid and Interface Science, vol. 170, no. 12, pp. $2-27,2012$

${ }^{44}$ Z. W. Ulissi, M. S. Strano, and R. D. Braatz, "Control of nano and microchemical systems," Computers \& Chemical Engineering, vol. 51, no. 0, pp. $149-156,2013$. \{CPC $\{$ VIII $\}$.

${ }^{45}$ H.-D. Yu, M. D. Regulacio, E. Ye, and M.-Y. Han, "Chemical routes to top-down nanofabrication," Chemical Society Reviews, vol. 42, no. 14, pp. 6006-6018, 2013.

${ }^{46} \mathrm{~V}$. N. Truskett and M. P. Watts, "Trends in imprint lithography for biological applications," Trends in Biotechnology, vol. 24, no. 7, pp. $312-317,2006$.

${ }^{47}$ B. C. Gross, J. L. Erkal, S. Y. Lockwood, C. Chen, and D. M. Spence, "Evaluation of $3 \mathrm{~d}$ printing and its potential impact on biotechnology and the chemical sciences," Analytical Chemistry, vol. 86, no. 7, pp. 3240-3253, 2014.

${ }^{48}$ Y. Xia, B. Gates, Y. Yin, and Y. Lu, "Monodispersed colloidal spheres: Old materials with new applications," Advanced Materials, vol. 12, no. 10, pp. 693-713, 2000.

${ }^{49}$ M. Grzelczak, J. Vermant, E. M. Furst, and L. M. Liz-Marzan, "Directed Self-Assembly of Nanoparticles," ACS Nano, vol. 4, pp. 3591-3605, JUL 2010.

${ }^{50}$ A. van Blaaderen, M. Dijkstra, R. van Roij, A. Imhof, M. Kamp, B. W. Kwaadgras, T. Vissers, and B. Liu, "Manipulating the self assembly of colloids in electric fields," European Physical Journal-Special Topics, vol. 222, pp. 2895-2909, NOV 2013.

${ }^{51}$ Y. Cui, M. Bjork, J. Liddle, C. Sonnichsen, B. Boussert, and A. Alivisatos, "Integration of colloidal nanocrystals into lithographically patterned devices," Nano Letters, vol. 4, pp. 10931098, JUN 2004.

${ }^{52}$ J. Cheng, C. Ross, H. Smith, and E. Thomas, "Templated selfassembly of block copolymers: Top-down helps bottom-up," Advanced Materials, vol. 18, no. 19, pp. 2505-2521, 2006.

${ }^{53}$ B. Gates, Q. Xu, M. Stewart, D. Ryan, C. Willson, and G. Whitesides, "New approaches to nanofabrication: Molding, printing, and other techniques," Chemical Reviews, vol. 105, pp. 1171-1196, APR 2005.

${ }^{54}$ P. Colson, C. Henrist, and R. Cloots, "Nanosphere Lithography: A Powerful Method for the Controlled Manufacturing of Nanomaterials," Journal of Nanomaterials, 2013.

${ }^{55}$ J. Y. Cheng, C. T. Rettner, D. P. Sanders, H.-C. Kim, and W. D. Hinsberg, "Dense self-assembly on sparse chemical patterns: Rectifying and multiplying lithographic patterns using block copolymers," Advanced Materials, vol. 20, no. 16, pp. 31553158, 2008.

${ }^{56}$ J. K. W. Yang, Y. S. Jung, J.-B. Chang, R. A. Mickiewicz, A. Alexander-Katz, C. A. Ross, and K. K. Berggren, "Complex self-assembled patterns using sparse commensurate templates with locally varying motifs.," Nature Nanotechnology, vol. 5, no. 4, pp. $256-260,2010$.

${ }^{57}$ A. F. Hannon, K. W. Gotrik, C. A. Ross, and A. AlexanderKatz, "Inverse design of topographical templates for directed self-assembly of block copolymers," ACS Macro Letters, vol. 2, no. 3, pp. 251-255, 2013.

${ }^{58}$ A. F. Hannon, Y. Ding, W. Bai, C. A. Ross, and A. AlexanderKatz, "Optimizing topographical templates for directed selfassembly of block copolymers via inverse design simulations," Nano Letters, vol. 14, no. 1, pp. 318-325, 2014.

${ }^{59}$ J.-B. Chang, H. K. Choi, A. F. Hannon, A. Alexander-Katz, C. A. Ross, and K. K. Berggren, "Design rules for self-assembled block copolymer patterns using tiled templates," Nature communications, vol. 5, 2014.

${ }^{60}$ J. Qin, G. S. Khaira, Y. Su, G. P. Garner, M. Miskin, H. M. Jaeger, and J. J. de Pablo, "Evolutionary pattern de- sign for copolymer directed self-assembly," Soft Matter, vol. 9, pp. 11467-11472, 2013.

${ }^{61} \mathrm{~S}$. Glotzer, M. Solomon, and N. A. Kotov, "Self-assembly: From nanoscale to microscale colloids," AIChE Journal, vol. 50, no. 12 , pp. $2978-2985,2004$

${ }^{62} \mathrm{~S}$. Glotzer and M. Solomon, "Anisotropy of building blocks and their assembly into complex structures," Nature Materials, vol. 6, no. 8, pp. 557-562, 2007.

${ }^{63}$ Y. Xia, Y. Xiong, B. Lim, and S. E. Skrabalak, "ShapeControlled Synthesis of Metal Nanocrystals: Simple Chemistry Meets Complex Physics?," Angewandte Chemie-International Edition, vol. 48, no. 1, pp. 60-103, 2009.

${ }^{64}$ P. F. Damasceno, M. Engel, and S. C. Glotzer, "Predictive self-assembly of polyhedra into complex structures," Science, vol. 337, no. 6093, pp. 453-457, 2012.

${ }^{65}$ U. Agarwal and F. A. Escobedo, "Mesophase behaviour of polyhedral particles," Nature Materials, vol. 10, pp. 230-235, MAR 2011.

${ }^{66}$ Z. Quan, H. Xu, C. Wang, X. Wen, Y. Wang, J. Zhu, R. Li, C. J. Sheehan, Z. Wang, D.-M. Smilgies, Z. Luo, and J. Fang, "Solvent-mediated self-assembly of nanocube superlattices," Journal of the American Chemical Society, vol. 136, no. 4, pp. 1352-1359, 2014.

${ }^{67}$ S. Sacanna, D. J. Pine, and G.-R. Yi, "Engineering shape: the novel geometries of colloidal self-assembly," Soft Matter, vol. 9, no. 34, pp. 8096-8106, 2013.

${ }^{68}$ C. B. Murray, C. Kagan, and M. Bawendi, "Synthesis and characterization of monodisperse nanocrystals and close-packed nanocrystal assemblies," Annual Review of Materials Science, vol. 30 , no. 1 , pp. $545-610,2000$.

${ }^{69}$ E. V. Shevchenko, D. V. Talapin, N. A. Kotov, S. O'Brien, and C. B. Murray, "Structural diversity in binary nanoparticle superlattices," Nature, vol. 439, no. 7072, pp. 55-59, 2006.

${ }^{70}$ D. J. Kraft, R. Ni, F. Smallenburg, M. Hermes, K. Yoon, D. A. Weitz, A. van Blaaderen, J. Groenewold, M. Dijkstra, and W. K. Kegel, "Surface roughness directed self-assembly of patchy particles into colloidal micelles," Proceedings of the $\mathrm{Na}$ tional Academy of Sciences, vol. 109, pp. 10787-10792, JUL 3 2012.

${ }^{71}$ P. Akcora, H. Liu, S. K. Kumar, J. Moll, Y. Li, B. C. Benicewicz, L. S. Schadler, D. Acehan, A. Z. Panagiotopoulos, V. Pryamitsyn, V. Ganesan, J. Ilavsky, P. Thiyagarajan, R. H. Colby, and J. F. Douglas, "Anisotropic self-assembly of spherical polymer-grafted nanoparticles," NATURE MATERIALS, vol. 8, pp. 354-U121, APR 2009.

${ }^{72}$ G.-R. Yi, D. J. Pine, and S. Sacanna, "Recent progress on patchy colloids and their self-assembly," Journal of PhysicsCondensed Matter, vol. 25, MAY 152013.

${ }^{73}$ T. Lafitte, S. K. Kumar, and A. Z. Panagiotopoulos, "Selfassembly of polymer-grafted nanoparticles in thin films," Soft Matter, vol. 10, pp. 786-794, 2014.

${ }^{74}$ G. Doppelbauer, E. G. Noya, E. Bianchi, and G. Kahl, "Selfassembly scenarios of patchy colloidal particles," Soft Matter, vol. 8, no. 30, pp. 7768-7772, 2012.

${ }^{75}$ E. Jankowski and S. C. Glotzer, "Screening and designing patchy particles for optimized self-assembly propensity through assembly pathway engineering," Soft Matter, vol. 8, no. 10, pp. 2852-2859, 2012.

${ }^{76}$ F. Romano and F. Sciortino, "Patterning symmetry in the rational design of colloidal crystals," Nature Communications, vol. 3, p. $975,2012$.

${ }^{77}$ J. D. Halverson and A. V. Tkachenko, "DNA-programmed mesoscopic architecture," Physical Review E, vol. 87, JUN 17 2013.

${ }^{78}$ D. Sun and O. Gang, "DNA-Functionalized Quantum Dots: Fabrication, Structural, and Physicochemical Properties," Langmuir, vol. 29, pp. 7038-7046, JUN 112013.

${ }^{79}$ I. Saaem and T. H. LaBean, "Overview of DNA origami for molecular self-assembly," Wiley Interdisciplinary Reviews Nanomedicine \& Nanobiotechnology, vol. 5, pp. 150-162, MAR- 
APR 2013.

${ }^{80}$ E. Auyeung, T. I. N. G. Li, A. J. Senesi, A. L. Schmucker, B. C. Pals, M. O. de la Cruz, and C. A. Mirkin, "DNAmediated nanoparticle crystallization into Wulff polyhedra," Nature, vol. 505, pp. 73-77, Jan 22014.

${ }^{81}$ Y. Wang, Y. Wang, D. R. Breed, V. N. Manoharan, L. Feng, A. D. Hollingsworth, M. Weck, and D. J. Pine, "Colloids with valence and specific directional bonding," Nature, vol. 491, pp. 51-U61, Nov 12012.

${ }^{82}$ M. G. Moffitt, "Self-assembly of polymer brush-functionalized inorganic nanoparticles: From hairy balls to smart molecular mimics," The Journal of Physical Chemistry Letters, vol. 4, pp. 3654-3666, 2013.

${ }^{83}$ L. Hong, A. Cacciuto, E. Luijten, and S. Granick, "Clusters of charged janus spheres," Nano Letters, vol. 6, no. 11, pp. 25102514, 2006.

${ }^{84} \mathrm{~S}$. Torquato, "Inverse optimization techniques for targeted selfassembly," Soft Matter, vol. 5, pp. 1157-1173, 2009.

${ }^{85}$ A. Jain, J. R. Errington, and T. M. Truskett, "Inverse design of simple pairwise interactions with low-coordinated 3D lattice ground states," Soft Matter, vol. 9, no. 14, pp. 3866-3870, 2013.

${ }^{86}$ E. Marcotte, F. H. Stillinger, and S. Torquato, "Communication: Designed diamond ground state via optimized isotropic monotonic pair potentials," The Journal of Chemical Physics, vol. 138 , no. 6 , p. $061101,2013$.

${ }^{87}$ E. Edlund, O. Lindgren, and M. N. Jacobi, "Using the uncertainty principle to design simple interactions for targeted selfassembly," The Journal of Chemical Physics, vol. 139, no. 2, p. $024107,2013$.

${ }^{88}$ G. Zhang, F. H. Stillinger, and S. Torquato, "Probing the limitations of isotropic pair potentials to produce ground-state structural extremes via inverse statistical mechanics," Physical Review E, vol. 88, no. 4, p. 042309, 2013.

${ }^{89}$ P. Schapotschnikow, R. Pool, and T. J. H. Vlugt, "Molecular simulations of interacting nanocrystals," Nano Letters, vol. 8, no. 9, pp. 2930-2934, 2008.

${ }^{90}$ M. I. Bodnarchuk, M. V. Kovalenko, W. Heiss, and D. V. Talapin, "Energetic and entropic contributions to self-assembly of binary nanocrystal superlattices: Temperature as the structuredirecting factor," Journal of the American Chemical Society, vol. 132 , no. 34 , pp. 11967-11977, 2010.

${ }^{91}$ A. Jain, J. R. Errington, and T. M. Truskett, "Communication: Phase behavior of materials with isotropic interactions designed by inverse strategies to favor diamond and simple cubic lattice ground states," The Journal of Chemical Physics, vol. 139, no. 14, p. 141102, 2013.

${ }^{92}$ D. Frenkel and A. J. C. Ladd, "New Monte Carlo method to compute the free energy of arbitrary solids. Application to the fcc and hcp phases of hard spheres," The Journal of Chemical Physics, vol. 81, no. 7, pp. 3188-3193, 1984.

${ }^{93}$ K. S. Rane, S. Murali, and J. R. Errington, "Monte Carlo simulation methods for computing liquid-vapor saturation properties of model systems," The Journal of Chemical Theory and Computation, vol. 9, no. 6, pp. 2552-2566, 2013.

${ }^{94}$ N. J. Fernandes, H. Koerner, E. P. Giannelis, and R. A. Vaia, "Hairy nanoparticle assemblies as one-component functional polymer nanocomposites: Opportunities and challenges," MRS Communications, vol. 3, no. 01 .

${ }^{95}$ J. Choi, C. M. Hui, M. Schmitt, J. Pietrasik, S. Margel, K. Matyjazsewski, and M. R. Bockstaller, "Effect of polymergraft modification on the order formation in particle assembly structures," Langmuir, vol. 29, no. 21, pp. 6452-6459, 2013.

${ }^{96}$ B. Srinivasan, T. Vo, Y. Zhang, O. Gang, S. Kumar, and V. Venkatasubramanian, "Designing DNA-grafted particles that self-assemble into desired crystalline structures using the genetic algorithm," Proceedings of the National Academy of Sciences, vol. 110, pp. 18431-18435, NOV 122013.

${ }^{97}$ R. J. Macfarlane, B. Lee, M. R. Jones, N. Harris, G. C. Schatz, and C. A. Mirkin, "Nanoparticle Superlattice Engineering with DNA," Science, vol. 334, pp. 204-208, OCT 142011.

${ }^{98}$ B. Vincent, J. Edwards, S. Emmett, and A. Jones, "Depletion flocculation in dispersions of sterically-stabilised particles (soft spheres)," Colloids and Surfaces, vol. 18, no. 24, pp. 261-281, 1986.

${ }^{99}$ E. B. Zhulina, O. V. Borisov, and V. A. Priamitsyn, "Theory of steric stabilization of colloid dispersions by grafted polymers," Journal of Colloid and Interface Science, vol. 137, no. 2, pp. 495 - 511, 1990.

${ }^{100}$ E. Currie, W. Norde, and M. C. Stuart, "Tethered polymer chains: surface chemistry and their impact on colloidal and surface properties," Advances in Colloid and Interface Science, vol. 100102, no. 0, pp. $205-265,2003$.

${ }^{101}$ D. J. Milliron, R. Buonsanti, A. Llordes, and B. A. Helms, "Constructing Functional Mesostructured Materials from Colloidal Nanocrystal Building Blocks," Accounts of Chemical Research, vol. 47, pp. 236-246, JAN 212014.

${ }^{102}$ R. Buonsanti and D. J. Milliron, "Chemistry of Doped Colloidal Nanocrystals," Chemistry of Materials, vol. 25, pp. 1305-1317, APR 232013.

${ }^{103}$ F. Felgner, L. Exel, M. Nesarajah, and G. Frey, "ComponentOriented Modeling of Thermoelectric Devices for Energy System Design," IEEE Transactions on Industrial Electronics, vol. 61, pp. 1301-1310, MAR 2014.

${ }^{104}$ G. Goel, W. P. Krekelberg, J. R. Errington, and T. M. Truskett, "Tuning density profiles and mobility of inhomogeneous fluids," Phys. Rev. Lett., vol. 100, p. 106001, Mar 2008.

${ }^{105}$ J. Carmer, G. Goel, M. J. Pond, J. R. Errington, and T. M. Truskett, "Enhancing tracer diffusivity by tuning interparticle interactions and coordination shell structure," Soft Matter, vol. 8, pp. 4083-4089, 2012.

${ }^{106}$ J. A. Millan, D. Ortiz, G. van Anders, and S. C. Glotzer, "Selfassembly of archimedean tilings with enthalpically and entropically patchy polygons," ACS Nano, vol. 0 , no. 0 , p. null, 0 .

${ }^{107}$ A. Facchetti, "Organic Semiconductors Made to order," Nature Materials, vol. 12, pp. 598-600, JUL 2013.

${ }^{108}$ Y. Diao, B. C.-K. Tee, G. Giri, J. Xu, D. H. Kim, H. A. Becerril, R. M. Stoltenberg, T. H. Lee, G. Xue, S. C. B. Mannsfeld, and Z. Bao, "Solution coating of large-area organic semiconductor thin films with aligned single-crystalline domains," Nature Materials, vol. 12, pp. 665-671, JUL 2013.

${ }^{109}$ M. Z. Miskin and H. M. Jaeger, "Adapting granular materials through artificial evolution," Nature Materials, vol. 12, pp. 326331, APR 2013.

${ }^{110}$ J. J. de Pablo and F. A. Escobedo, "Molecular simulations in chemical engineering: Present and future," AIChE Journal, vol. 48 , no. 12 , pp. 2716-2721, 2002.

${ }^{111}$ I. Grossmann and L. Biegler, "Part II. Future perspective on optimization," Computers \& Chemical Engineering, vol. 28, pp. 1193-1218, JUL 152004.

${ }^{112}$ B. Hartke, "Global optimization," Wiley Interdisciplinary Reviews - Computational Molecular Science, vol. 1, pp. 879-887, NOV-DEC 2011.

${ }^{113}$ M.-H. Lin, J.-F. Tsai, and C.-S. Yu, "A Review of Deterministic Optimization Methods in Engineering and Management," Mathematical Problems in Engineering, 2012.

${ }^{114}$ W. Paszkowicz, "Genetic Algorithms, a Nature-Inspired Tool: A Survey of Applications in Materials Science and Related Fields: Part II," Materials and Manufacturing Processes, vol. 28, pp. 708-725, JUL 32013. 